\title{
Tuberculosis care among refugees arriving in Europe: a ERS/WHO Europe Region survey of current practices
}

\author{
Masoud Dara ${ }^{1,15}$, Ivan Solovic ${ }^{2,15}$, Giovanni Sotgiü3,15, Lia D’Ambrosio ${ }^{4,5,15}$, \\ Rosella Centis ${ }^{4,15}$, Richard Tran ${ }^{1,15}$, Delia Goletti ${ }^{6}$, Raquel Duarte ${ }^{7}$, \\ Stefano Aliberti ${ }^{8}$, Fernando Maria de Benedictis ${ }^{9}$, Graham Bothamley ${ }^{10}$, \\ Tom Schaberg $^{11}$, Ibrahim Abubakar ${ }^{12}$, Vitor Teixeira ${ }^{13}$, Brian Ward ${ }^{13}$, \\ Christina Gratziou ${ }^{14}$ and Giovanni Battista Migliori ${ }^{4}$
}

Affiliations: ${ }^{1}$ World Health Organization Office at the European Union, Brussels, Belgium. ${ }^{2}$ National Institute for TB, Lung Diseases and Thoracic Surgery, Vysne Hagy, Catholic University Ruzomberok, Ruzomberok, Slovakia. ${ }^{3}$ Clinical Epidemiology and Medical Statistics Unit, Dept of Biomedical Sciences, University of Sassari - Research, Medical Education and Professional Development Unit, AOU Sassari, Sassari, Italy. ${ }^{4}$ WHO Collaborating Centre for TB and Lung Diseases, Fondazione S. Maugeri IRCCS, Tradate, Italy. ${ }^{5}$ Public Health Consulting Group, Lugano, Switzerland. ${ }^{6}$ Translational Research Unit, Epidemiology Dept, National Institute for Infectious Diseases, Rome, Italy. ${ }^{7}$ EPI Unit, Institute of Public Health, University of Porto, Porto, Portugal. ${ }^{8}$ School of Medicine and Surgery, University of Milan-Bicocca, UO Clinica Pneumologica, AO San Gerardo, Monza, Italy. ${ }^{9}$ Dept of Pediatrics, Salesi Children's Hospital Foundation, Ancona, Italy. ${ }^{10}$ Dept of Respiratory Medicine, Homerton University Hospital NHS Foundation Trust, London, UK. ${ }^{11}$ Dept of Pneumology, Diaconess Hospital Rotenburg/Wümme, Rotenburg/Wümme, Germany. ${ }^{12}$ Institute for Global Health, University College London, London, UK. ${ }^{13}$ European Respiratory Society, Brussels Office, Brussels, Belgium. ${ }^{14}$ University Respiratory Medicine Unit, Evgenidio Hospital, Athens, Greece. ${ }^{15}$ These authors contributed equally.

Correspondence: Giovanni Battista Migliori, World Health Organization Collaborating Centre for Tuberculosis and Lung Diseases, Fondazione S. Maugeri, Care and Research Institute, Via Roncaccio 16, 21049, Tradate, Italy. E-mail: giovannibattista.miglioriafsm.it

ABSTRACT No evidence exists on tuberculosis (TB) and latent TB infection (LTBI) management policies among refugees in European countries.

A questionnaire investigating screening and management practices among refugees was sent to 38 national TB programme representatives of low and intermediate TB incidence European countries/ territories of the WHO European Region.

Out of 36 responding countries, 31 (86.1\%) reported screening for active TB, 19 for LTBI, and eight (22.2\%) reporting outcomes of LTBI treatment. Screening for TB is based on algorithms including different combinations of symptom-based questionnaires, bacteriology and chest radiography and LTBI screening on different combinations of tuberculin skin test and interferon- $\gamma$ release assays. In 22 (61.1\%) countries, TB and LTBI screening are performed in refugee centres. In $22(61.1 \%)$ countries, TB services are organised in collaboration with the private sector. 27 (75\%) countries answered that screening for TB is performed as per national and international guidelines, while $19(52.7 \%)$ gave the same answer with regards to LTBI screening. Infection control measures are inadequate in several of the countries surveyed.

There is need for improved coordination of TB screening in Europe to implement the End TB Strategy and achieve TB elimination.

$@$ ERSpublications

TB/LTBI detection and management in refugees in low/intermediate incidence European countries: policies and practice http://ow.ly/aNx8301yx3j

This article has supplementary material available from erj.ersjournals.com

Received: April 282016 | Accepted after revision: June 102016 | First published online: Aug 042016

Conflict of interest: V. Teixeira and B. Ward are employees of the European Respiratory Society.

Copyright OERS 2016 


\section{Introduction}

According to the most recent World Health Organization (WHO) Global TB Report the estimated annual tuberculosis (TB) incidence decreased globally by an average of $1.5 \%$ per year since 2000 and the estimated TB prevalence in 2014 was $42 \%$ lower than in 1990 [1]. However, an estimated 9.6 million people worldwide developed active TB in 2014, among them, 12\% had HIV infection [1]. During the same year, TB caused 1.5 million deaths, making it one of the most common causes of death from an infectious disease alongside HIV.

In 2014, a total of 329270 TB cases were reported from 51 countries in the WHO European Region (notification rate: 36.7 cases per 100000 population), with 33000 estimated deaths [2]. The estimated incidence in Europe represents 3\% of the global TB burden.

TB is considered a major public health challenge in many countries worldwide, particularly among vulnerable populations, such as individuals at higher risk of exposure to discrimination, hostility or economic adversity. These factors unfortunately afflict the lives of many migrants and refugees (here defined in agreement with the 1951 "Convention and Protocol relating to the status of Refugees": www.unhcr.org/3b66c2aa10.html) [1-5].

Several factors have contributed to increase population mobility in the WHO European Region, such as the establishment of the European Union (EU) and free movement within the Newly Independent States (NIS), particularly for seasonal labour [5, 6], although refugees' problems are specific. This increased population mobility poses challenges for $\mathrm{TB}$ control and requires effective and sustainable mechanisms to ensure quality TB and latent TB infection (LTBI) prevention, diagnosis and treatment $[5,7]$.

The need for coordinated intervention in these areas is justified from the perspective of individual human rights (independent of legal or residential status of the subject) as well as public health pre-requisites to control and ultimately eliminate TB, including multi- and -extensively drug resistant TB (MDR-TB and XDR-TB) [3, 8-10].

For refugees, full access to TB diagnosis and treatment (with guarantee of protection from deportation until the end of treatment) has been recommended by WHO; this is in the interest of both the individual and the wider hosting community in terms of TB control and elimination [11-15]. In 2015, more than one million migrants and refugees reached Europe by land and sea. In 2014 the estimated figure was significantly lower (219000) [4, 16]. According to official data, an estimated 181673 new refugees and migrants arrived in Europe between January 1 and April 26, 2016, with 1261 reported deaths. 82\% of arrivals via the Mediterranean sea originated from 10 countries only [16]. At the current time, the four countries from which most refugees originate are the Syrian Arab Republic (43\% of the overall flow), Afghanistan (23\%), Iraq (14\%), Pakistan (4\%) and Iran (4\%) (see also: http://ec.europa.eu/eurostat/statistics-explained/index.php/File: First_time_asylum_applicants_in_the_EU-28_by_citizenship,_Q4_2014_\%E2\%80\%93_Q4_2015.png).

Despite the recent release of resolutions and statements by bodies such as WHO, the European Respiratory Society (ERS) and the EU [4, 17], not much is known about the policies in force in European countries with regards to TB and LTBI management among refugees upon arrival.

In light of the ongoing refugee situation in Europe, the aim of this ERS/WHO European Region study (performed through the ERS ad hoc Working Group on TB Advocacy) is to document the policies and practices of low and intermediate TB incidence European countries with regards to detection and management of TB and LTBI among refugees.

\section{Methods}

\section{Survey questionnaire}

In September-October 2015, experts from the ERS, WHO Regional Office for Europe and the WHO Collaborating Centre in Tradate, Italy, as members of the ERS ad hoc Working Group on TB Advocacy, (www.ersnet.org/index.php?option=com_flexicontent\&view=items\&id=5200-tb-advocacy-working-group.

html) developed a short questionnaire for a rapid survey containing multiple choice and open-ended questions on screening and management of TB and LTBI among refugees in Europe. The questionnaire was finalised after reviewing suggestions and comments received from the members of the ERS TB Advocacy ad hoc Working Group and reaching overall consensus among the members. In addition to basic demographic data of the respondents, the survey comprised questions on the following subject areas: screening for and management of TB/LTBI; guidelines, legislation and evidence for current practice; cross-border TB care; and organisational aspects of TB care and infection control measures.

The questionnaire was sent to the national TB programme representatives of all EU/European Economic Area countries of the WHO European Region, Switzerland and six other countries who have hosted, or were deemed likely to host, or become a transient country for a significant number of refugees in the near future. The six additional countries were the current EU candidate countries (Albania, Bosnia and Herzegovina, the former Yugoslav Republic of Macedonia, Montenegro, Serbia and Turkey). The survey, along with a cover letter for additional information, was sent to each of the national TB programme representatives on October 
23, 2015, with an initial deadline set for November 6, 2015. Furthermore, there was an offer for the TB programme representatives to conduct a telephone interview to complete the survey, should returning the document prove too difficult by the deadline provided. On November 9, 2015, a reminder email was sent to programme representatives who had not responded. The survey was closed on February 24, 2016.

\section{Data analysis}

The results of the survey were entered into a Microsoft Excel programme (Excel 2010; Microsoft Corporation, Albuquerque, NM, USA) and double-checked (L. D'Ambrosio; R. Centis) prior to analysis. Results produced a mixture of quantitative and qualitative data, with descriptive statistics being calculated where appropriate, and supplemented with qualitative information provided by responders to the survey.

\section{Ethics}

As a broad evaluation of current policies and practices within countries, ethical approval was not required because the study did not collect individualised information on subjects.

\section{Results}

$36(94.7 \%)$ out of 38 countries contacted responded to the questionnaire. The results from section 1 (Screening for TB and LTBI among refugees in the European Region) are summarised in table 1.

Refugees are routinely screened for active TB by the majority of the countries (31 (86.1\%) out of 36), with the exception of Italy, Monaco and Portugal where a non-systematic screening is performed (only in symptomatic individuals); no screening is performed in Former Yugoslavia Republic of Macedonia (length of stay in holding centre is not long enough for screening to take place) and Serbia (insufficient governmental funding). 19 (52.7\%) countries (Belgium, Bulgaria, Croatia, Cyprus, Estonia, France, Greece, Iceland, Lithuania, Luxembourg, Malta, Montenegro, Norway, Romania, Slovakia, Spain, Sweden, Turkey and the UK) screen systematically for LTBI among refugees, eight (22.2\%) countries (Denmark, Finland, Germany, Italy, Monaco, the Netherlands, Portugal and Slovenia (which reported low numbers)) do not perform it systematically and nine (25\%) do not screen at all for LTBI (Albania, Austria, Czech Republic, Hungary, Ireland, Latvia, Former Yugoslav Republic of Macedonia, Serbia and Switzerland) (figure 1).

However, almost half of the countries (8 (47\%) out of 17$)$ that currently do not screen for TB and LTBI have plans to introduce it for TB and/or LTBI in the near future. There is a legal obligation to screen for TB and/or LTBI in 21 (58.3\%) of the 36 countries responding to this survey.

Screening for $\mathrm{TB}$ is performed with algorithms using different combinations of symptom-based questionnaires (21 (58.3\%) out of 36 , of which one not systematically collected), bacteriology (18 (50\%) out of 36, sputum smear/culture collection of which nine for symptomatic individuals only) and chest radiography (27 $(75 \%)$ out of 36 , of which two do not perform systematically, while in Germany, TB screening of adult immigrants ( $\geqslant 15$ years old) is almost systematically performed by chest radiography (with exception of pregnant ladies as per legal act)); five countries (Denmark, Italy, Monaco, Portugal and Turkey) do not systematically perform any TB-specific examination. In two countries (Croatia and Hungary), routine bacteriology for TB (sputum smear and culture) is part of the screening procedure.

Similar to the findings described by a previous ERS/WHO Europe Region Study [10], LTBI screening is performed by using different combinations of tuberculin skin test (TST) and interferon- $\gamma$ release assays (IGRAs) in $23(63.8 \%)$ out of 36 different European countries (eight (22.2\%) out of 36, TST only, 11 $(30.5 \%)$ out of 36 , TST plus IGRA, four $(11.1 \%)$ out of 36 , TST plus IGRA in selected cases (only in Bacillus Calmette-Guérin non-vaccinated children aged $<12$ years and after TB exposure in the Netherlands; and in case of recent exposure to TB in Portugal, Slovenia and Monaco).

In $22(61.1 \%)$ out of 36 countries, TB and LTBI screening are performed in refugee centres, using also other combinations of measures (See table 1 for details). The decision to perform TB/LTBI screening is determined by the TB incidence rate in the country of origin of refugees in $14(38.8 \%)$ out of 36 of the surveyed countries. No single threshold was provided. In the majority of countries where any screening takes place, it is performed only once (28 (90.3\%) out of 31 ). The results from section 2 (Management of $\mathrm{TB}$ and LTBI among refugees in Europe) are summarised in table 2.

In the majority of countries $(24(66.6 \%)$ out of 36$)$ treatment after diagnosis of active TB in a refugee is required, whereas in Denmark, Monaco, the Netherlands and Portugal, TB treatment is voluntary; in six other countries (Belgium, Germany, Ireland, Sweden, Switzerland and the UK) individuals cannot be legally forced to take medications, but can usually be convinced to start anti-TB treatment; involuntary isolation is foreseen in case of refusal to comply with treatment, while in Serbia isolation is only considered for MDR-TB patients who refuse treatment and, in Macedonia, no TB treatment is proposed due to the short length of stay in the country. Overall, no EU country reported that TB detection was a reason for deportation. 
TABLE 1 Questionnaire section I: screening for tuberculosis and latent tuberculosis infection among refugees in Europe

Active tuberculosis screening

Yes: $31 / 36(86.1 \%)$

No: $2 / 36(5.2 \%)$

Not systematically: $3 / 36(8.3 \%)$

Latent tuberculosis infection screening

Yes: $19 / 36(52.7 \%)$

No: $9 / 36(25 \%)$

Not systematically: $8 / 36$ (22.2\%)

Plans to implement screening for active tuberculosis/latent tuberculosis infection Yes: $8 / 17(47 \%)^{\#}$

No: $5 / 17(29.4 \%)^{\#}$

Not answered: $3 / 17(17.6 \%)^{\#}$

Not applicable: $1 / 17(5.9 \%)^{\#}$

Legal requirement for screening

Yes: $21 / 36(58.3 \%)$

No: $12 / 36(33.3 \%)$

Not applicable: $3 / 36$ (13.8\%)

Active tuberculosis screening

Yes, systematic symptoms-based questionnaires: $20 / 36$ (55.5\%)

Yes, not systematic symptoms-based questionnaire: $1 / 36(2.7 \%)$

Yes, systematic bacteriology: 9/36 (25\%)

Yes, bacteriology for symptomatic individuals only: 9/36 (25\%)

Yes, systematic chest radiography: $25 / 36(69.4 \%)^{\pi}$

Yes, not systematic chest radiography: $2 / 36(5.5 \%)$

Other procedures: $1 / 36(2.7 \%)^{+}$

Not systematic screening for active tuberculosis: $5 / 36(13.9 \%)^{\S}$

Routine sputum collection for microbiological study/culture/Xpert

Yes: $2 / 36(5.5 \%)$

No: $34 / 36(94.4 \%)$

Latent tuberculosis infection screening

Yes, tuberculin skin test: 19/36 (52.7\%)

Yes, tuberculin skin test only: $8 / 36$ (22.2\%)

Yes, tuberculin skin test plus interferon- $\gamma$ release assays: $11 / 36$ (30.5\%)

Yes, not systematic tuberculin skin test plus interferon- $\gamma$ release assays: $4 / 36$ (11.1\%)

Not applicable for both: $13 / 36(36.1 \%)$

Place of screening

Refugee centres only: $10 / 36(27.7 \%)$

On arrival only: $4 / 36$ (11.1\%)

In the community only: $1 / 36(2.7 \%)$

In the National Tuberculosis Programme Centre only: 1/36 (2.7\%)

On arrival and in refugee centres: $6 / 36(16.6 \%)$

On arrival and at pre-arrival: $1 / 36(2.7 \%)$

On arrival and in the community: 1/36 (2.7\%)

On pre-arrival and in the community: $1 / 36(2.7 \%)$

In refugee centres and in the community: $6 / 36$ (16.6\%)

No applicable information: $5 / 36$ (13.8\%)

Information on tuberculosis rates in the country of origin to decide for the screening process Yes: $14 / 36(38.8 \%)$

No: $19 / 36(52.7 \%)$

Not applicable: $3 / 36$ (13.8\%)

Screening number

Only once: $28 / 31(90.3 \%)^{f}$

More than once: $3 / 31(9.7 \%)^{f}$

\#: denominator is the number of countries that do not (or do not systematically) screen for tuberculosis/ latent tuberculosis infection; " : in Germany, tuberculosis screening almost systematically performed by chest radiography for adult immigrants $(\geqslant 15 \text { years old })_{;}{ }^{+}$: initial algorithm with tuberculin skin tests and blood examination; ${ }^{\S}$ : numerator includes countries which do not systematically perform any examination; $f$ : denominator is the number of countries that screen for tuberculosis/latent tuberculosis infection.

Anti-TB treatment is proposed immediately after diagnosis in the majority of countries (26 (72.2\%) out of $36)$, where costs are covered by central governmental funds (26 (72.2\%) out of 36). Almost three-quarters (23 $(63.8 \%)$ out of 36$)$ report that efforts are ongoing to adapt TB services to refugees' specific needs 


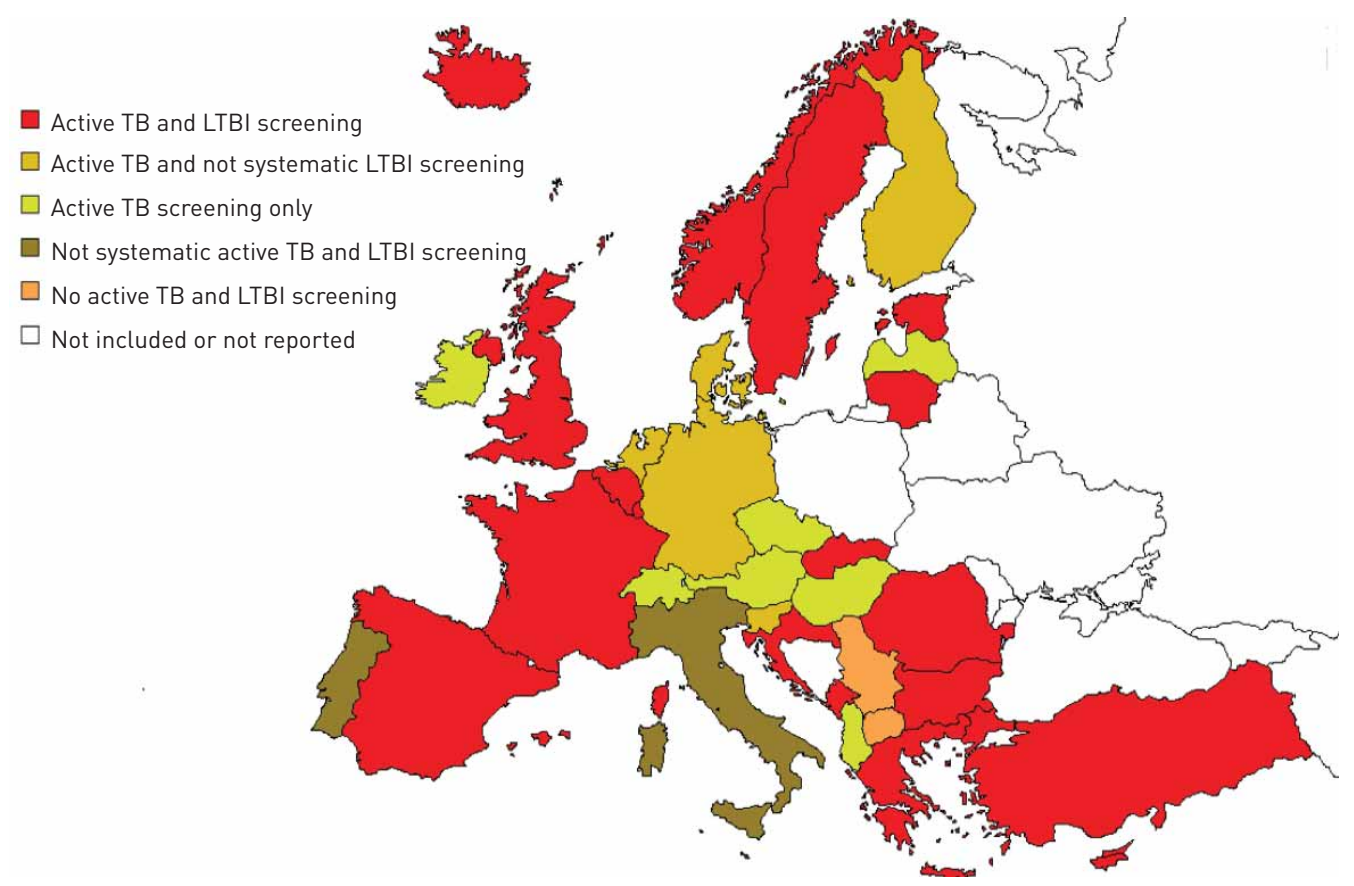

FIGURE 1 Summary of the countries screening for tuberculosis (TB) and latent TB infection (LTBI).

through specific national/regional programmes and improved cooperation with the non-governmental sector. A similar number of countries (22 (61.1\%) out of 36), directly or indirectly (through certified non-governmental organisations (NGOs)) allow refugees access to TB services. Among countries with general or specific regional/national programmes (or guidelines) for TB management in refugees (14 (38.8\%) out of 36), more than half (nine (64.2\%) out of 14) report difficulties in fully complying with requirements of their own guidelines, given the high number of refugees in the present situation. Further details on specific national programmes are available in table 2 .

The results from section 3 (Guidelines, Legislation and Evidence on the results of screening and treatment of TB and LTBI in Europe) are summarised in table 3. In particular, 27 (75\%) 36 countries answered that screening for $\mathrm{TB}$ is done as per national and international guidelines (offering the same services to refugees and nationals), while 19 (52.7\%) out of 36 gave the same answer with regards to LTBI screening. Similarly, while $22(61.1 \%)$ out of 36 countries confirmed that they collect data on the yield of active TB screening among refugees (with Estonia, Finland, Norway and the UK partially/not systematically collecting data), only 11 (30.5\%) out of 36 countries (Bulgaria, Finland, France, Iceland, Italy, Lithuania, Norway, Slovakia, Slovenia, Turkey and the UK) are equipped to collect similar data for LTBI screening (Finland, Norway and the UK providing data not systematically). Finally, detailed information on TB treatment outcomes is available in $19(52.7 \%)$ out of 36 countries, while treatment completion rates for LTBI therapy among refugees are available in only eight $(22.2 \%)$ countries (Bulgaria, France, Iceland, Netherlands, Portugal, Slovakia and Slovenia and Turkey).

The results from section 4 (Organisational aspects of TB care and infection control issues) are summarised in table 4. Seven (19.4\%) countries (Austria, Croatia, Germany, Greece, the Former Yugoslavia Republic of Macedonia, Serbia and Turkey) reported to host $>250000$ refugees in the 6 months preceding the survey, Hungary notified a range between 100001 and 250000, while Italy and Sweden reported hosting between 50001 and 100000 refugees. In the vast majority of the countries (30 (83.3\%) out of 36), public sector services are in charge of managing refugees for TB-related issues, complemented by international organisations (e.g. Red Cross in Bulgaria, Denmark, the Former Yugoslavia Republic of Macedonia, Serbia and Spain; the International Organization for Migration in Romania and Medicine du Monde in the UK).

Several problems were reported among the different countries, including internal and external communication and coordination issues, cultural mediation/language differences and inadequate funding or human resources. The sheer volume of refugees was also cited as a challenge in eight $(22.2 \%)$ countries (Austria, Belgium, Germany, Greece, Italy, the Netherlands, Norway, Serbia). Although respirators are generally available to protect staff and complement administrative infection control measures, a general lack of consistency with international guidelines emerged from the countries' answers. 
TABLE 2 Questionnaire section II: management of tuberculosis and latent tuberculosis infection among refugees in Europe

\section{Procedures if active tuberculosis is diagnosed}

No: refusal of asylum: 34/36 (94.4\%)

Yes: obligation to undergo treatment: $24 / 36(66.6 \%)$

Other: $10 / 36(27.7 \%)$

Not applicable: $2 / 36$ (5.5\%)

Obligation to undergo treatment

Where

Treatment in hospital: $24 / 36(66.6 \%)$

Not applicable: $8 / 36(22.2 \%)$

Not answered: $4 / 36$ (11.1\%)

When

Treatment immediately started after diagnosis: $26 / 36$ (72.2\%)

Not answered 2/36 (5.5\%)

Not applicable: $8 / 36$ (22.2\%)

Funding

Governmental funds: $26 / 36$ (72.2\%)

Not answered: $2 / 36$ (5.5\%)

Not applicable: $8 / 36(22.2 \%)$

Procedures if latent tuberculosis infection is diagnosed

No, refusal of asylum: 20/36 (55.5\%)

Yes, obligation to undergo preventive therapy: $8 / 36$ (22.2\%)

Other: 18/36 (50\%)

Not applicable: $8 / 36$ (22.2\%)

Obligation to undergo preventive therapy

Proposed to all positive for latent tuberculosis infection

Proposed to all positive for latent tuberculosis infection: $3 / 36$ (8.3\%)

No, proposed for specific groups and ages only: 7/36 (19.4\%)

Not applicable: $24 / 36$ (66.6\%)

Not answered: $1 / 36(2.7 \%)$

Same procedure as native nationals positive for latent tuberculosis infection

Same procedure as native nationals positive for latent tuberculosis infection: $7 / 36(19.4 \%)$

Not applicable: $24 / 36(66.6 \%)$

Not answered: $5 / 36$ (13.8\%)

Therapy delivery

Therapy delivered at chest/directly observed treatment/tuberculosis centres/tuberculosis specialists: $7 / 36(19.4 \%)$

Not applicable: $23 / 36(63.8 \%)$

Not answered: $6 / 36(16.6 \%)$

Funding

Government budget: $9 / 36$ (25\%)

Not applicable: $23 / 36$ (63.8\%)

Not answered: $4 / 36$ (11.1\%)

Regional/national specific programmes for tuberculosis management in refugees

Yes: $10 / 36(27.7 \%)$

No, not fully specific: $4 / 36(11.1 \%)$

No: $22 / 36$ (61.1\%)

Regional/national programmes to provide sensitive services

Yes: $23 / 36(63.8 \%)$

No: $1 / 36(2.7 \%)$

Not answered: 12/36 (33.3\%)

Special measures to deal with undocumented migrants

Yes: $22 / 36(61.1 \%)$

No: $1 / 36(2.7 \%)$

Not answered: $13 / 36$ (36.1\%)

Discrepancy/ies between guidelines and implementation

Yes: $9 / 36(25 \%)$

No: $6 / 36(16.6 \%)$

Not answered: $8 / 36(22.2 \%)$

Not applicable: $13 / 36$ (36.1\%)

Tuberculosis management funding

Government funds: 22/36 (61.1\%)

Not answered: 12/36 (33.3\%)

Not applicable: 2/36 (5.5\%) 
TABLE 3 Questionnaire section III: guidelines, legislation and evidence on the results of screening and treatment of tuberculosis and latent tuberculosis in Europe

\section{Screening and management of active tuberculosis among refugees according to national or} international guidelines/legislation in force

Yes: $27 / 36(75 \%)$

No: $3 / 36(8.3 \%)$

Not applicable: $1 / 36(2.7 \%)$

Not answered: $5 / 36(13.8 \%)$

Screening and management of latent tuberculosis infection among refugees according to national or international guidelines/legislation in force

Yes: $19 / 36(52.7 \%)$

No: $7 / 36(19.4 \%)$

Not applicable: $5 / 36(13.8 \%)$

Not answered: $5 / 36(13.8 \%)$

Data collection in place to assess the yield of screening for active tuberculosis among refugees

Yes: $18 / 36(50 \%)$

Yes, partially or not systematically: $4 / 36$ (11.1\%)

No: $8 / 36(22.2 \%)$

Not answered: $4 / 36(11.1 \%)$

Not applicable: $2 / 36(5.5 \%)$

Data collection in place to assess the yield of screening for latent tuberculosis infection among

refugees

Yes: $8 / 36(22.2 \%)$

Yes, partially or not systematically: $3 / 36$ (8.3\%)

No: $18 / 36$ (50\%)

Not answered: $4 / 36(11.1 \%)$

Not applicable: $3 / 36$ (8.3\%)

Data collection in place to assess treatment success rates of active tuberculosis among refugees Yes: $19 / 36(52.7 \%)$

No: $10 / 36(27.7 \%)$

Not answered: $6 / 36(16.6 \%)$

Not applicable: $1 / 36$ (2.7\%)

Data collection in place to assess completion rates of latent tuberculosis among refugees

Yes: $8 / 36(22.2 \%)$

No: $20 / 36(55.5 \%)$

Not answered: $6 / 36(16.6 \%)$

Not applicable: $2 / 36$ (5.5\%)

\section{Discussion}

The aim of our study was to investigate which reported policies and practices exist for TB and LTBI screening and management among refugees in low and intermediate TB incidence countries of Europe. The survey had a very high response rate (36 (94.7\%) out of 38) which shows countries' interest and prioritisation of this issue.

The results of our study confirm that screening for TB is considered as an important public health measure in Europe, although significant differences exist in screening practices among countries.

According to a survey conducted in 2012 on screening practices on infectious diseases among newly arrived migrants to Europe, all countries perform TB screening, with the second-most screened condition being Hepatitis B (30\% of the countries) [18]. The results of our survey also indicate that there is a general lack of analysis of the yield of TB and LTBI screening among refugees. The huge workload is assumed as the main reason. Furthermore, much less information is available for LTBI than for active TB disease.

While our survey shows that 31 countries regularly screen refugees for TB, only 19 screen for LTBI, and even a fewer report outcomes of LTBI treatment [9]. In view of TB elimination, more and more emphasis will be given to the possibility of implementing LTBI registers in low TB incidence countries [19].

Although 11 out of 36 countries answered they were equipped to collect information on LTBI screening, only three (Finland, Norway and the UK) confirmed collection of data, although not systematically. LTBI management remains a component of the TB Elimination strategy which is challenging countries, particularly when a large number of patients is involved. In fact, the large number of arrivals in holding centres, particularly in some European countries, makes LTBI screening and subsequent management problematic. In addition, several countries reported difficulties in coordination between holding centres and TB services serving the native population. 
TABLE 4 Questionnaire section IV: organisational aspects of tuberculosis care and infection control issues

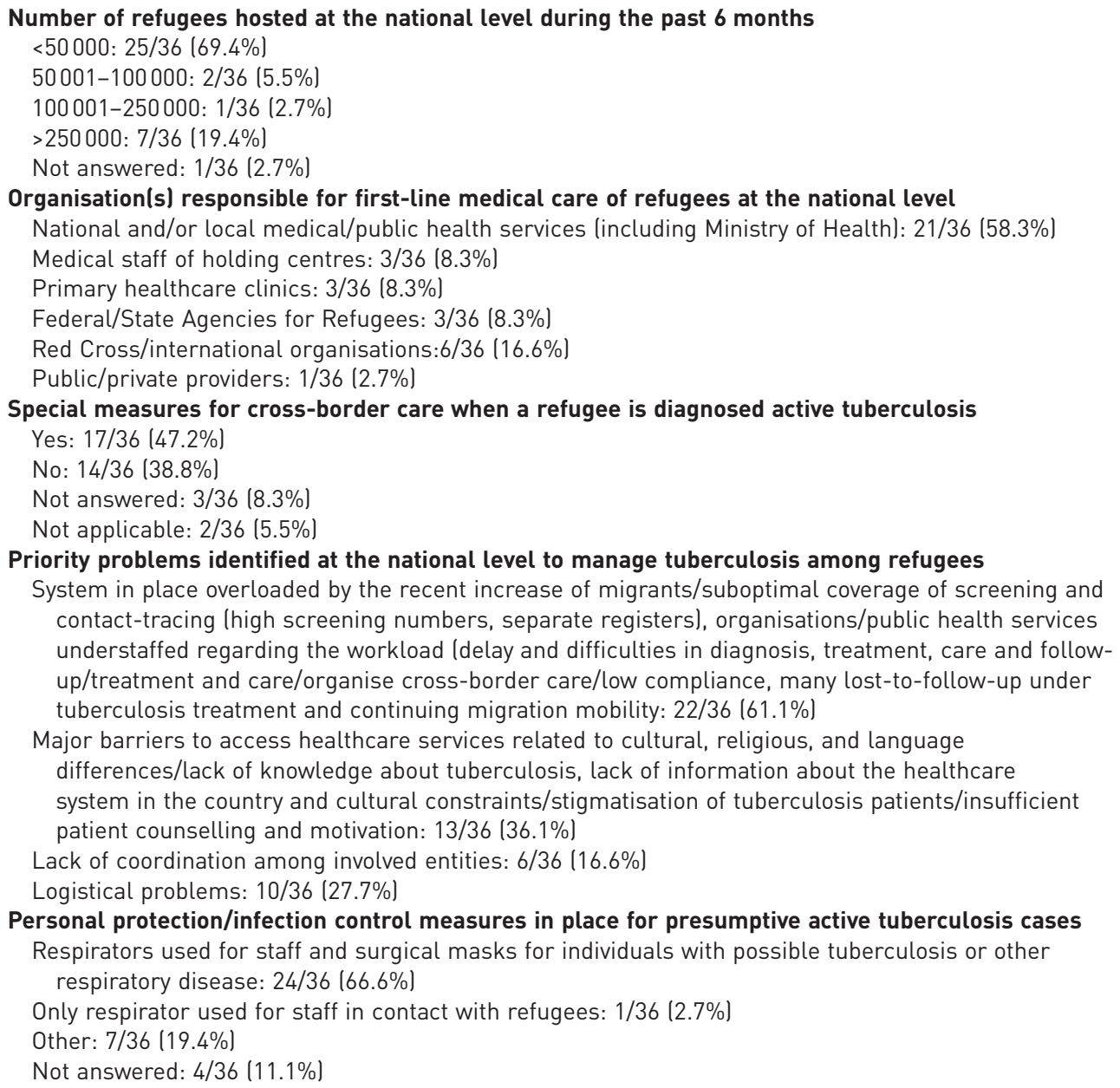
contact-tracing (high screening numbers, separate registers), organisations/public health services understaffed regarding the workload (delay and difficulties in diagnosis, treatment, care and followup/treatment and care/organise cross-border care/low compliance, many lost-to-follow-up under tuberculosis treatment and continuing migration mobility: 22/36 (61.1\%)

Major barriers to access healthcare services related to cultural, religious, and language differences/lack of knowledge about tuberculosis, lack of information about the healthcare system in the country and cultural constraints/stigmatisation of tuberculosis patients/insufficient patient counselling and motivation: 13/36 (36.1\%)

Lack of coordination among involved entities: $6 / 36$ (16.6\%)

Logistical problems: 10/36 (27.7\%)

Personal protection/infection control measures in place for presumptive active tuberculosis cases

Respirators used for staff and surgical masks for individuals with possible tuberculosis or other respiratory disease: $24 / 36(66.6 \%)$

Only respirator used for staff in contact with refugees: $1 / 36(2.7 \%)$

Other: $7 / 36(19.4 \%)$

Not answered: $4 / 36(11.1 \%)$

Based on our survey, it appears that there are no systematic follow-up screening/check-ups of refugees for TB sometime after their arrival. Given that refugees are often exposed to precarious, stressful travelling conditions during transit, which provide a risk of Mycobacterium tuberculosis transmission, there is a need to ensure people-centred care is available to them beyond arrival in their host country. Symptomatic screening of refugees and more intensive follow-up for those with LTBI may be justified. This is particularly important as many European countries are scaling-up their efforts to eliminate TB [10].

In an attempt to make screening as cost effective as possible, countries have applied different algorithms in-line with WHO recommendations [20]. According to our survey, they are based on different combinations of symptom-baseds and chest radiography, with addition of bacteriology in a few countries (table 2). Evaluation of the yield of these screening procedures was beyond the scope of this study; however, there is a real need for analysis of such data at national and regional level $[5,6]$.

Our study identified different models of screening for TB/LTBI in Europe. Some countries perform radiological screening of all refugees in a hub or holding centre, and carry out further investigations in decentralised centres only if radiological abnormalities are identified. Others implement different screening algorithms or organise provision of health services differently at the refugee centres. In this context, screening for LTBI, by use of IGRAs and/or TST, although considered an important intervention in the pursuit of TB Elimination $[9,10]$ is still difficult to implement in several countries.

Based on the unprecedented number of refugees, it is important to have a specific response plan and ensure its full funding both at the national and European level. Interestingly, in the majority of countries (22 (61.1\%) out of 36), TB services are organised in collaboration with NGOs and other sectors. Among others benefits, this approach has the advantage of increasing cultural sensitivity of the TB services. 
Infection-control measures are generally inadequate in a large proportion of the countries surveyed. Surgical masks are often used to "protect" healthcare workers when it is well known that they are ineffective against M. tuberculosis from active TB patients who are not on treatment; certified respirators are needed for this purpose. Furthermore, a lack of specific training on infection control measures has been reported. Similar problems have previously been described in European MDR-TB reference centres [21].

Although surveys of this kind are subject to several limitations (related to the instrument used, the missing information from non-responding countries, the possible erroneous responses from national programmes and the limitations of aggregated data), strengths of this study include: continuous dialogue with National TB representatives; the very high response rate (94.7\%); and the consistency of the answers received with previous surveys carried out using similar methodology [10].

The results of the present study highlight the need for improved coordination of TB screening in Europe, with the ultimate goal of implementing the End TB Strategy, the TB Action Plan for the WHO European Region 2016-2020 [22] and the Health 2020 Policy Framework [23] to address inequity. The ultimate goal of these strategies is to achieve TB Elimination $[9,10,24,25]$. This will require quality operational research evaluating surveillance (aimed at attaining better data for better planning), the efficacy of existing algorithms and the yield of screening activities [26]. Furthermore, within the limited information available on LTBI in the European context, further clinical and operational research is also needed to inform clinicians and public health authorities on the correct approach to follow when LTBI is diagnosed in contacts of MDR-TB cases.

Finally, the new function of the ERS/WHO Europe TB Consilium (a free, internet-based instrument supporting clinicians to manage difficult-to-treat cases of tuberculosis) is now live and accessible under the TB Consilium website (www.tbconsilium.org). This electronic platform will allow better cross-border TB control by contributing to the provision of quality prevention, diagnostic and treatment services to migrants and refugees.

\section{Acknowledgements}

The Authors alone are responsible for the views expressed in this publication and they do not necessarily represent the decisions and policies of their Institutions.

The National representatives who contributed to collect the survey data were: Donika Mema Bardhi (Albania); Alina Virsa (Austria); Maryse Wanlin and Wouter Arrazola de Oñate (Belgium); Mariya Zamfirova (Bulgaria); Aleksandar Simunovic (Croatia); Constantia Voniatis (Cyprus); Peter Henrik Andersen and Annette Hartvig Christiansen (Denmark); Piret Viiklepp, Manfred Danilovitš (Estonia); Hanna Soini (Finland); Thierry Comolet (France); Barbara Hauer (Germany); Ourania Kalkouni (Greece); Gábor Kovács (Hungary); Joan O Donnell and Sarah Jackson (Ireland); Thorsteinn Blöndal (Iceland); Maria Grazia Pompa and Francesco Paolo Maraglino (Italy); Irina Lucenko (Latvia); Edita Davidaviciene (Lithuania); Pierre Weicherding (Luxembourg); Biljana Ilievska Poposka (Republic of Macedonia); Analita Pace-Asciak (Malta); Jean Lorenzi (Monaco); Olivera Bojovic and Stevan Lucic (Montenegro); Gerard de Vries (the Netherlands); Trude Arnesen and Karine Nordstrand (Norway); Raquel Duarte (Portugal); Georgeta Gilda Popescu and Chiotan Domnica Ioana (Romania); Violeta Mihailovic-Vucinic (Serbia); Ivan Solovic (Slovakia); Petra Svetina (Slovenia); Elena Andradas Aragonés (Spain); Jerker Jonsson (Sweden); Peter Helbling (Switzerland); Erhan Kabasakal (Turkey); Dominik Zenner and Alison Smith-Palmer (UK).

\section{References}

1 World Health Organization. Global tuberculosis control 2015. Document WHO/HTM/TB/2015.22. Geneva, World Health Organization, 2015.

2 European Centre for Disease Prevention and Control/WHO Regional Office for Europe. Tuberculosis surveillance and monitoring in Europe 2016. Stockholm, European Centre for Disease Prevention and Control, 2016.

3 International Organisation for Migration (IOM). International Migration Law №25 - Glossary on Migration. 2nd Edn. Geneva, IOM, 2011. www.epim.info/wp-content/uploads/2011/01/iom.pdf Date last accessed: April 26, 2016.

4 Matteelli A, Lönnroth $\mathrm{K}$, Mosca $\mathrm{D}$, et al. Cameroon's multidrug-resistant tuberculosis treatment programme jeopardised by cross-border migration. Eur Respir J 2016; 47: 686-688.

5 Dara M, Solovic I, Goletti D, et al. Preventing and controlling tuberculosis among refugees in Europe: more is needed. Eur Respir J 2016; 48: 272-274.

6 de Vries G, van Rest J, Meijer W, et al. Low yield of screening asylum seekers from countries with a tuberculosis incidence of less than 50 per 100.000 population. Eur Respir J 2016; 47: 1870-1872.

7 Dara M, de Colombani P, Petrova-Benedict R, et al. The minimum package for cross-border TB control and care in the WHO European region: a Wolfheze consensus statement. Eur Respir J 2012; 40: 1081-1090.

8 Dara M, Kluge H. Roadmap to prevent and combat drug-resistant tuberculosis. Copenhagen, World Health Organization, Regional Office for Europe, 2011. www.euro.who.int/_data/assets/pdf_file/0014/152015/e95786.pdf Date last accessed: February 23, 2016.

9 Lönnroth K, Migliori GB, Abubakar I, et al. Towards tuberculosis elimination: an action framework for low-incidence countries. Eur Respir J 2015; 45: 928-952.

10 D'Ambrosio L, Dara M, Tadolini M, et al. Tuberculosis elimination: theory and practice in Europe. Eur Respir J 2014; 43: 1410-1420.

11 Recommendations to ensure the diagnosis and treatment of tuberculosis in undocumented migrants. International Union against Tuberculosis and Lung Disease, 2008. www.theunion.org/get-involved/join-theunion/body/RESS_ Undocumented-migrants-Statement_2008.pdf Date last accessed: February 23, 2016. 
12 Migliori GB, Zellweger JP, Abubakar I, et al. European Union standards for tuberculosis care. Eur Respir J 2012; 39: $807-819$

13 van der Werf MJ, Sandgren A, D'Ambrosio L, et al. The European Union standards for tuberculosis care: do they need an update? Eur Respir J 2014; 43: 933-942.

14 Migliori GB, Sotgiu G, D'Ambrosio L, et al. TB and MDR/XDR-TB in the EU and EEA countries: managed or mismanaged? Eur Respir J 2012; 39: 619-625.

15 Veen J, Migliori GB, Raviglione MC, et al. Harmonisation of TB control in the WHO European region: the history of the Wolfheze workshops. Eur Respir J 2011; 37: 950-959.

16 UNHCR - The UN Refugee Agency. Refugees and migrants crossing the Mediterranean to Europe. Overview of arrival trends as of April 12, 2016. http://data.unhcr.org/mediterranean/regional.php Date last accessed: April 12, 2016.

17 World Health Organization Sixty-Seventh World Health Assembly. Global strategy and targets for tuberculosis prevention, care and control after 2015. A67/11. Geneva, World Health Organization, 2014. http://apps.who.int/ gb/ebwha/pdf_files/WHA67/A67_11-en.pdf Date last accessed: April 26, 2016.

18 Napoli C, Dente MG, Kärki T, et al. Screening for infectious diseases among newly arrived migrants: experiences and practices in non-EU countries of the Mediterranean basin and Black Sea. Int J Environ Res Public Health 2015; 12: 15550-8.

19 World Health Organization. Report of the Global Consultation on the Programmatic Management of Latent Tuberculosis Infection. 27-28 April 2016, Seoul, Republic of Korea. Document WHO/HTM/TB/2016.08. Geneva, World Health Organization, 2016.

20 World Health Organization. Systematic screening for active tuberculosis: principles and recommendations. Document WHO/HTM/TB/2013.04. Geneva, World Health Organization, 2013.

21 Sotgiu G, D'Ambrosio L, Centis R, et al. TB and M/XDR-TB infection control in European TB reference centres: the Achilles' heel? Eur Respir J 2011; 38: 1221-1223.

22 World Health Organization Regional Office for Europe. Tuberculosis action plan for the WHO European Region 20162020. www.euro.who.int/en/about-us/governance/regional-committee-for-europe/65th-session/documentation/workingdocuments/eurrc6517-rev.1-tuberculosis-action-plan-for-the-who-european-region-20162020 Date last accessed: April 26, 2016.

23 World Health Organization Regional Office for Europe Health 2020: the European policy for health and well-being. www.euro.who.int/en/health-topics/health-policy/health-2020-the-european-policy-for-health-and-wellbeing/about-health-2020 Date last accessed: April 26, 2016

24 Diel R, Loddenkemper R, Zellweger JP, et al. Old ideas to innovate tuberculosis control: preventive treatment to achieve elimination. Eur Respir J 2013; 42: 785-801.

25 Uplekar M, Weil D, Lonnroth K, et al. WHO’s Global TB Programme. WHO’s new End TB Strategy. Lancet 2015; 385: 1799-1801.

26 Bothamley GH, Ditiu L, Migliori GB, et al. Active case-finding in Europe: a TBNET (Tuberculosis Network European Trials group) survey. Eur Respir J 2008; 31: 1023-1030. 